\title{
Correlative Characteristics of Leakage and Structural Factors in Multistage Soft Plunger Pump
}

\author{
Hu Chunying ${ }^{1}$, Han Guoyou ${ }^{2, *}$, Yu Haiquan ${ }^{1}$ and Ye Weidong ${ }^{2,3}$ \\ ${ }^{1}$ College of Engineering, Heilongjiang Bayi Agricultural University, Daqing 163319, China \\ ${ }^{2}$ School of Mechanical Science and Engineering, Northeast Petroleum University, Daqing 163318, China \\ ${ }^{3}$ Khartoum Refinery Co. Ltd, Khartoum North 12211, Sudan
}

Received 4 December 2020; Accepted 20 February 2021

\begin{abstract}
Soft plunger pumps have attracted the attention of many oil fields in China and other countries due to their advantages, such as their anti-scaling feature, simple structure, and convenient maintenance. The soft plunger pump has good adaptability to the well conditions of alkaline surfactant polymer (ASP) flooding technology. However, the short inspection period of soft plunger pumps in production hinders their further popularization and limits their application. To explore the relationship between the leakage rate of the pump and the structural parameters, a vertical annular slit flow model of a multistage soft plunger and a pump barrel was proposed. Using Lagrange-Euler to describe the problem of the interface displacement between fluid and solid, the deformation of the soft plunger was solved in an iterative manner, and the deformation cloud diagram and leakage distribution of the multistage soft plunger pump were obtained. On the basis of the fluid mechanics and the law of mass conservation, the relevant factors affecting leakage were analyzed, including the length and thickness of the soft plunger and the initial clearance between the soft plunger and pump barrel pair. The influences of these factors on leakage were explored by combining theoretical analysis and numerical simulation. Results show that under different initial clearance conditions of the soft plunger and pump barrel pair, the leakage of the oil pump is negatively correlated with the length of the soft plunger, that is, the longer the soft plunger is, the smaller the leakage will be. An exponential relationship exists between leakage and the thickness of the soft plunger. The leakage increases with the initial clearance between the soft plunger and pump barrel pair, and the relationship between the leakage of the oil pump and the initial clearance of the soft plunger-pump barrel pair is approximately at third power. Under the condition of satisfying the conservation of mass, from the fluid inlet to the fluid outlet, the length of the soft plunger decreases and the thickness and the initial clearance between the soft plunger and pump barrel pair increase. This study provides a theoretical basis for improving the pumping efficiency of the soft plunger pump and optimizing the structural parameters of the soft plunger.
\end{abstract}

Keywords: Soft plunger pump, Leakage amount, Alkaline surfactant polymer, Pumping efficiency

\section{Introduction}

With the increasing difficulty of oil exploitation, alkaline surfactant polymer(ASP) flooding technology has been adopted in some oil fields with alkali, polymer solution, and surfactant as driving fluids, increasing the oil recovery rate by more than $20 \%$. However, the addition of polymers causes some mineral components to dissolve and migrate, produces an aluminum silicate mixture between the plunger of the pump and the pump barrel, and even results in sand cards and fouling in severe cases. During the peak period of fouling, the pump inspection cycle is greatly shortened to just over 70 days. At present, two methods are usually used to solve the fouling problem of the ASP flooding well. The period of pump inspection can be extended using the chemical anti-scale and non-chemical anti-scale methods. For example, the single-stage hydraulic self-sealing soft plunger pump is a production device based on metal-nonmetal surface contact. The device operates with the threephase mixed medium of crude oil, water, and natural gas

*E-mail address: hchunying@163.com

ISSN: 1791-2377 @ 2021 School of Science, IHU. All rights reserved. doi:10.25103/jestr.141.22 under medium and high temperatures, and some results have been achieved in solving the fouling problem of conventional plunger pumps.

On this basis, the soft plunger material, leakage, and lifting performance of oil well pumps were studied in [1-3]. Then, the soft plunger materials were optimized, and the relationship between leakage and the lifting performance is revealed. However, under two-way fluid-solid coupling, the influence of the leakage rate has been rarely studied, resulting in the deviation of the physical model and leakage rate of the soft plunger pump from the actual working state. Thus, the problems of pump efficiency and life span cannot be solved correctly by existing studies. Therefore, the related characteristics of leakage volume and structural factors of multi-stage soft plunger pumps must be urgently studied to build an accurate predictive model of soft plunger pumps and clarify the coupling relationship between the fluid and the soft plunger in the gap between the soft plunger and pump barrel pair in actual operation.

For the single-stage plunger pump to bear low pressure and to prolong the service life of the soft plunger pump, a multi-stage soft plunger pump adapted to ASP flooding technology is designed to achieve uniform pressure in each stage. To predict a more accurate functional relationship 
between the working parameters and leakage amount of soft plunger pumps, a soft plunger pump model is set up using the finite element analysis method and the leakage situation are analyzed under different soft plunger pump lengths and initial gap cases. Then, on the basis of the model and the analysis, a reference for the optimization of soft plunger pump parameters is provided.

\section{State of the art}

Several soft plunger pumps have been developed and applied in oil fields around the world. According to statistics, the service life of soft seal plunger pumps is $55 \%-60 \%$ longer than ordinary pumps [4]. Scholars have conducted many works on soft plunger pumps. Given the serious scaling of the string of ASP flooding wells and the frequent pumping of pumping units, Ren et al. developed a multistage soft plunger pump and conducted pressure resistance and leakage tests. The average pump efficiency was $62.84 \%$, and the longest maintenance-free period was 184 days, effectively prolonging the pump inspection period and service life of oil wells. However, the preferred cup hydraulically swelled and was prone to wear when fitted with the plunger [5]. Wang and others studied a soft plunger fishing fixed valve oil pump through field wear, pressure, and leakage tests in Daqing Oilfield. The plunger of the pump was mainly made of fluoroplastic material and added with auxiliary materials. Pump inspection was conducted without moving the oil pipe, and the operation cost of the oil well was reduced. However, the pump was only suitable for oil wells with no or very little sand production [6]. Yang et al. compared the application effects of the ASP flooding lifting process system. The average inspection period of the 92-well multistage soft plunger pumping pumps used in the field was 215 days, which could effectively reduce the ternary combination flooding pump phenomenon, but the study did not discuss the soft plunger structure parameters and the pump efficiency comprehensively [7]. To reduce the abrasion of soft-sealed leather bowls, Cui et al. discussed the pressure transmission law of soft plunger pumps and matched the shape of the pressure relief groove with the angle of the intercepting pressure transmission hole to achieve a seal [8]. To address the shortcomings of traditional oil wells, such as sand-bearing wells, scaling in ASP flooding, and large leakage, Richardson developed a new combination of plunger anti-seize oil wells and effectively shortened the test period. To improve the pump inspection period, the high temperature resistance and anti-aging performance of the sealing cup must be improved and the service life of the soft plunger must be extended [9]. Lee analyzed the structural characteristics of the anti-seizure pump for ASP flooding, created a solid model of the antiseizure pump, and verified the model through wear resistance and leakage experiments on an oil pump. Given the complex working conditions in ASP flooding wells, the wear failure exposed during the work requires further exploration [10]. Newton carried out works on immersive thickening pumps. With hydraulic feedback force, immersive thickening pumps could change the pressure difference between the tubing and the casing into the downward force of the sucker rod string, which could address the difficult downward movement of the rod string in heavy oil wells. However, further work must be carried out on the checking of the sealing section of the thickening pump [11]. Jafri analyzed the status quo of ternary combined flooding pumping wells, developed a new type of pumping pump, which used a combination of segmented lifting technology and soft plunger technology, and conducted indoor simulation tests to verify if the performance satisfied the lifting requirements. Extending the inspection period of ASP flooding pumping wells had great significance, but the field application study for this new type of pump had not been combined with complex working conditions [12]. Zhao carried out indoor temperature change and abrasion tests on different soft plunger materials in response to the current limited pressure and wear resistances of polytetrafluoroethylene and nylon. Polyether ether ketone with high wear resistance and excellent chemical stability was preferred as the main sealing ring material for soft plunger sucker pumps. Field tests showed that soft plungers of this material could not only meet the requirements of underground work, but also effectively reduce the construction process and operating costs. However, the deformation behavior of polyether ether ketone soft plungers under the action of the flow field and its influence on the pressure distribution of the flow field must be discussed further [13]. Li et al. used the experimental comparison method to analyze the sensitivity of conventional and soft plunger segmented oil well pumps to the sand particles suspended in the pipe string and found that the soft plunger subsection pump operates smoothly in the presence of sand particles, that is, pump jamming caused by dirt or sand particles could be effectively prevented. However, the pump's low efficiency below kilometers limits its use [14]. Li used the orthogonal multi-parameter optimization design method to study the flexible oil pumping system of a plunger pump, developed an indoor test scheme, determined a reasonable pump clearance value through several optimization tests, and solved several problems, such as the swelling of the rubber sleeve and excessive pull-up load. However, the sealing and expansion ring materials of the hydraulic self-sealing soft plunger pump have poor wear resistance and a short service life [15]. Gao meshed and refined the three-dimensional model of the swash plate plunger pump based on Gambit. Using the UDF method in the Fluent software, the flow state of the plunger pump was analyzed, and the law of motion of the two-phase boundary was explored. However, the flow changes of the inlet and outlet of the plunger pump under the state of the convectionsolid coupling must be studied and analyzed in detail [16]. Kireev used the Fluent software to simulate the temperature, velocity, and other flow field changes in the oil pumping process of ordinary plunger pumps. The two-way fluidstructure coupling method was adopted to study the plunger and the interstitial oil film, but the leakage amount of the clearance oil film flow field was not calculated according to the simulated operating conditions of the soft plunger pump [17]. Ham discussed fluid-solid coupling under the different changes in structure parameters of the plunger sleeve and under the effect of the gap flow deformation characteristics, proposed a clearance self-compensation hydraulic pump plunger vice structure, by the theoretical analysis and experiment validation annular groove width increase with the increase of plunger sleeve deformation. However, under the same boundary conditions and work pressure, such as plunger sleeve deformation and material thickness, the relevant characteristics of the structural parameters must be studied [18]. Medvedovski carried out theoretical analysis and experimental work on plunger and ball valve movement. Using the Fluent software, the movement model of the plunger pump was constructed, and the changes in the 
plunger velocity and pressure fields during the upstroke were obtained. The analysis of the internal flow field characteristics provides a reference, but the internal flow field characteristics of the plunger under different operating conditions were not discussed [19].

The above studies focused mainly on the design method, structure, and temperature and velocity fields of soft plunger and few studies have been conducted on leakage characteristics, especially the correlation between leakage and the main structural parameters. In this study, the twoway fluid-solid coupling analysis method is used to establish a model of the working characteristics of the soft plunger pump, the vertical annular gap flow between the soft plunger and the pump barrel is investigated, and a mathematical model of leakage is built. From the internal flow characteristics of the pump in the beginning, the influence factors and their relationship to the leakage volume of the oil well pumps are discussed separately under different soft plunger lengths, thicknesses, and soft plungerpump barrel pair initial clearances, and a basis for the optimization and testing of soft plunger oil well pumps is provided.

The rest of this study is organized as follows. In the third section, a two-stage soft plunger pump is taken as an example to build a physical model. Through the two-way coupling between the soft plunger and the clearance flow in the pump barrel, the influencing factors of leakage and their functional relations are explored. In the fourth section, the leakage characteristics of the model are analyzed through the combination of numerical simulation and curve fitting, and the leakage characteristics under different structural parameters are obtained. Relevant conclusions are summarized in the last section.

\section{Methodology}

\subsection{Physical Model}

The structure of the two-stage soft plunger pump is shown in Fig. 1. The device adopts the principles of hydraulic selfsealing and grading pressure. The lower joint of the first stage is connected to the upper joint of the second stage, that is, the two-stage soft plunger pump is composed of a series of structures. When the plunger is in the upper stroke stage, the soft plunger swimming valve is closed, and the oil flows into the first stage soft plunger through the four through holes in the upper joint of the soft plunger, increasing the internal liquid pressure of the soft plunger. At this time, although the soft plunger is forced to expand, it is insufficient to fill the entire pump barrel. Therefore, the gap between the soft plunger and the pump barrel after expansion can be designed according to the requirements of different working conditions. The oil enters the second stage soft plunger through the gap between the first stage soft plunger and the pump barrel. However, due to the resistance along the way, a certain pressure drop occurs after the oil passes through the first stage soft plunger. The low-pressure oil continues to flow through the four through holes of the upper joint of the second stage soft plunger into the second stage soft plunger, resulting in the expansion and deformation of the second stage soft plunger. Therefore, the pressure decreases step by step, forming a multi-stage seal and grading pressure, so that each stage of the soft plunger is not subjected to excessive pressure difference, greatly improving the service life of the soft plunger.
When the soft plunger is in the downstroke stage, the swimming valve of the second stage soft plunger is turned on, and the oil in the pump barrel flows into the first stage plunger through the upper joint. Meanwhile, the swimming valve of the first stage soft plunger is turned on, so the pump barrel is connected up and down, and the pressure difference between the inside and outside of the soft plunger is equal, causing the soft plunger to shrink to its original shape and the downward resistance of the soft plunger to decrease. The structure ensures the lubrication between the friction pairs and is very suitable for heavy oil exploitation.

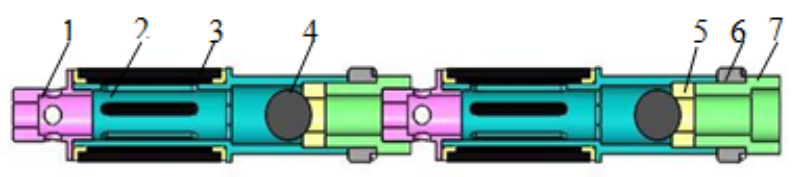

1 -On the joint; 2 -Supporting frame; 3 -Soft plunger; 4 -Traveling valve; 5 -Traveling valve seat; 6 -Centralizer; $7-$ The connectors Fig. 1. Structural diagram of two-stage soft plunger pump

\subsection{Influence of length on the leakage of soft plunger pump}

The leakage of the multi-stage soft plunger pump is a measure of the performance of the pump. The leakage of the soft plunger of the pump without relative movement is called static leakage, which is determined by the following equation:

$Q=300 \pi d h_{0}^{3} \Delta p / \mu l$

In the formula, $d$ is the outer diameter of the soft plunger, $\mathrm{m} ; h_{0}$ is the clearance between the soft plunger and the pump barrel after initial installation, $\mathrm{m} ; \Delta p$ is the pressure difference between both ends, $\mathrm{Pa} ; \mu$ is the hydrodynamic viscosity, Pa.s; $l$ is the length of the soft plunger, $\mathrm{m}$.

To carry out the structural design and parameter optimization of the multistage soft plunger pump and explore the pressure and velocity distribution of the multistage soft plunger, the relationship between the leakage of the multistage soft plunger pump and the influencing factors must be elucidated. Using the Fluent software to establish the soft computing model of the plunger, the leakage and the deformation are calculated under different soft plunger lengths. The liquid medium oil well pump outlet pressure of $2 \mathrm{MPa}$, the inlet pressure of $0 \mathrm{MPa}$, the pump barrel diameter of $30 \mathrm{~mm}$, the soft plunger thickness of 3 $\mathrm{mm}$, and soft plunger pump barrel-vice initial gaps of 0.4 , $0.45,0.5,0.55$, and $0.6 \mathrm{~mm}$, as shown in Tables 1 and 2 .

Table 1. Leakage of soft plunger with different lengths

\begin{tabular}{l|l|l|l|l|l}
\hline \multirow{2}{*}{$\begin{array}{l}\text { lengths } \\
l(\mathrm{~mm})\end{array}$} & \multicolumn{5}{|l}{ initial clearance $h_{0}(\mathrm{~mm})$} \\
\cline { 2 - 6 } & 0.4 & 0.45 & 0.5 & 0.55 & 0.6 \\
\cline { 2 - 6 } & leakage $Q(\mathrm{~kg} / \mathrm{s})$ & \\
\hline 30 & 0.00607 & 0.00900 & 0.01275 & 0.01275 & 0.02260 \\
35 & 0.00521 & 0.00771 & 0.01092 & 0.01092 & 0.01934 \\
40 & 0.00456 & 0.00674 & 0.00955 & 0.00955 & 0.01692 \\
45 & 0.00406 & 0.00628 & 0.00849 & 0.00849 & 0.01505 \\
50 & 0.00365 & 0.00540 & 0.00750 & 0.00750 & 0.01358 \\
\hline
\end{tabular}

The deformation in Table 2 refers to the maximum deformation of the soft plunger during the upper stroke. When the initial clearance is different, the maximum 
deformation of the soft plunger is slightly different. To determine the location of the maximum deformation of the soft plunger and explore the influence of the length of the soft plunger on the deformation under the same clearance, the deformation cloud diagram of the two-way fluidstructure coupling is calculated at the initial clearance of 0.4 $\mathrm{mm}$ and the lengths of 40, 45, and $50 \mathrm{~mm}$, as shown in Fig. 2. The figure shows that the deformation of the outer wall of the soft plunger increases gradually from top to bottom, and the maximum deformation is located at its lower part. The deformation cloud diagrams of the soft plunger calculated under different clearances and lengths all meet the change rule, mainly because the difference between the internal and external fluid loads on the lower part of the outer wall of the soft plunger during the upper stroke is larger than that on the upper part of the outer wall. The length of the soft plunger is one of the influencing factors of the amount of deformation. If the other parameters are kept as quantitative, the amount of deformation increases with the length of the soft plunger.

Table 2. Deformation of soft plunger with different lengths

\begin{tabular}{|c|c|c|c|c|c|}
\hline \multirow{3}{*}{$\begin{array}{l}\text { lengths } \\
l(\mathrm{~mm})\end{array}$} & \multicolumn{5}{|c|}{ initial clearance $h_{0}(\mathrm{~mm})$} \\
\hline & 0.4 & 0.45 & 0.5 & 0.55 & 0.6 \\
\hline & \multicolumn{5}{|c|}{ deformation $\chi(\mathrm{mm})$} \\
\hline 30 & 0.0890 & 0.0886 & 0.0885 & 0.0883 & 0.0880 \\
\hline 35 & 0.0903 & 0.0903 & 0.0901 & 0.0899 & 0.0895 \\
\hline 40 & 0.0916 & 0.0916 & 0.0913 & 0.0910 & 0.0906 \\
\hline 45 & 0.0927 & 0.0925 & 0.0923 & 0.0920 & 0.0915 \\
\hline 50 & 0.0935 & 0.0933 & 0.0930 & 0.0926 & 0.0923 \\
\hline
\end{tabular}

\section{D: Transient Structural Directional Deformation Unit: $\mathrm{mm}$ \\ Coordinate System \\ Time: 5.e-002 \\ $2020 / 3 / 22 \quad 18: 56$

\begin{tabular}{|l|l}
$\mathbf{0 . 0 9 1 5 7 4} \mathbf{M a x}$ \\
0.080837 \\
0.070101 \\
0.059364 \\
0.048627 \\
0.03789 \\
0.027153 \\
0.016416 \\
0.0056791 \\
$\mathbf{- 0 . 0 0 5 0 5 7 8 ~ M i n}$
\end{tabular}

Type: Directional Deformation(X Axis)

(a)

\begin{tabular}{|c|}
\hline 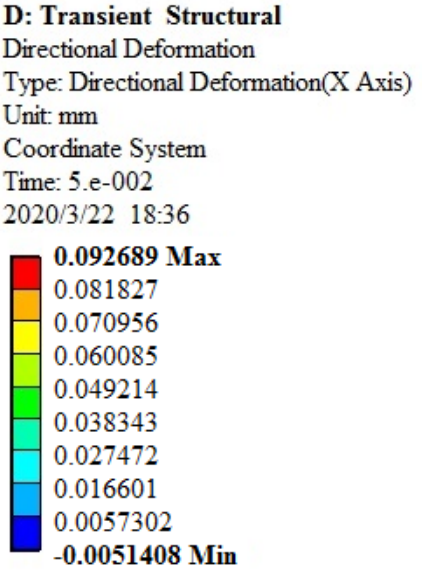 \\
\hline
\end{tabular}

(b)

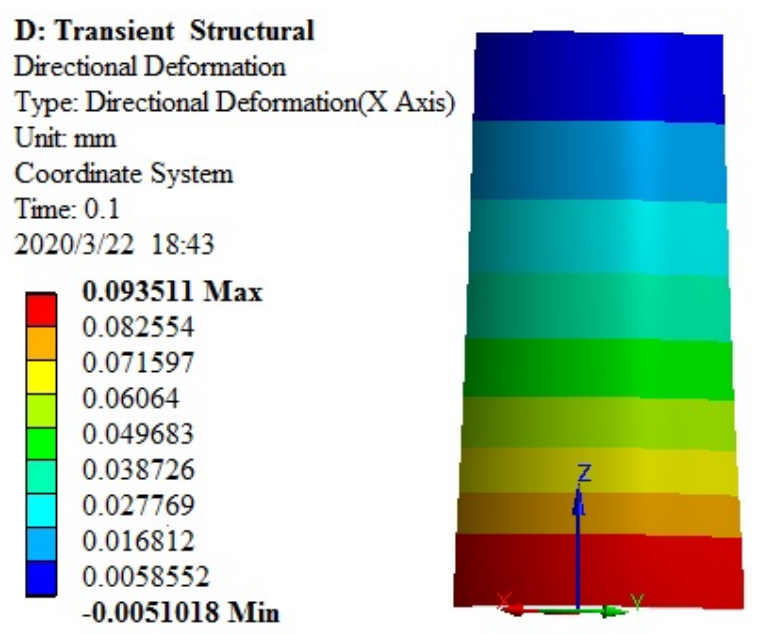

(c)

Fig. 2. Deformations of soft plunger of different lengths (a) $40 \mathrm{~mm}$ (b) $45 \mathrm{~mm}$ (c) $50 \mathrm{~mm}$

\subsection{Influence of thickness on the leakage of the soft plunger oil pump}

The deformation and leakage of the soft plunger during the up-stroke operation are affected by the thickness factor. To explore the relationship between the two, the outlet pressure of the oil pump is set to $2 \mathrm{MPa}$, and the inlet pressure is set to $0 \mathrm{MPa}$. The structural parameters of the soft plunger are as follows: the length is $50 \mathrm{~mm}$, and the initial clearance between the soft plunger and the pump barrel pair is $0.7 \mathrm{~mm}$. Under this condition, the deformation and leakage of the soft plunger with different thicknesses are calculated as shown in Table 3.

Table 3. Deformation and leakage of soft plunger with different thickness

\begin{tabular}{l|l|l|l}
\hline $\begin{array}{l}\text { thickness } \\
\delta(\mathrm{mm})\end{array}$ & $\begin{array}{l}\text { deformation } \\
\chi(\mathrm{mm})\end{array}$ & $\begin{array}{l}\text { stress } \\
(\mathrm{MPa})\end{array}$ & $\begin{array}{c}\text { leakage } \\
Q^{(\mathrm{kg} / \mathrm{s})}\end{array}$ \\
\hline 2.0 & 0.150 & 14.989 & 0.0195 \\
2.5 & 0.115 & 12.194 & 0.0211 \\
3.0 & 0.091 & 10.334 & 0.0222 \\
3.5 & 0.082 & 6.096 & 0.0231 \\
4.0 & 0.062 & 8.028 & 0.0237 \\
\hline
\end{tabular}

3.4 Influence of initial clearance on the leakage of soft plunger oil pump

The main factors affecting the clearance of the oil pump are downhole temperature, pressure, and the liquid gravity of the soft plunger, which cause the radial deformation of the soft plunger and pump barrel pair [21-22]. The length of the soft plunger is $50 \mathrm{~mm}$, and the thickness is $3 \mathrm{~mm}$. If the same pressure difference condition is met, differential pressure can be achieved by setting different outlet and inlet pressures. That is, the outlet pressure of the oil pump is 6,4 , and 2 $\mathrm{MPa}$, respectively, and the inlet pressure is 4,2 , and $0 \mathrm{MPa}$, respectively. The two-way fluid-structure coupling method is used to calculate the leakage and deformation of the soft plunger and pump barrel pair at different initial clearances, as shown in Table 4.

\section{Result Analysis and Discussion}

On the basis of the calculation model and numerical value of leakage in the two-way fluid-structure coupling in Sections $3.2-3.4$, the Matlab software is used to fit the leakage of the 
multi-stage soft plunger pump and explore the influence of the main structural parameters of soft plunger on the leakage of the pump. Theoretical support is provided for structural optimization and parameter design.

Table 4. Leakage and deformation of soft plunger under different initial clearances

\begin{tabular}{l|l|l|l|l|l|l}
\hline \multirow{2}{*}{$\begin{array}{l}\text { initial } \\
\text { clearance }\end{array}$} & \multicolumn{3}{|c|}{ leakage $Q\left(10^{-2} \mathrm{~kg} / \mathrm{s}\right)$} & \multicolumn{3}{|c}{ deformation $\chi(\mathrm{mm})$} \\
\cline { 2 - 7 }$h_{0}(\mathrm{~mm})$ & $6-4$ & $\begin{array}{l}4-2 \\
\mathrm{MPa}\end{array}$ & $\begin{array}{l}2-0 \\
\mathrm{MPa}\end{array}$ & $\begin{array}{l}6-4 \\
\mathrm{MPa}\end{array}$ & $\begin{array}{l}4-2 \\
\mathrm{MPa}\end{array}$ & $\begin{array}{l}2-0 \\
\mathrm{MPa}\end{array}$ \\
\hline 0.75 & 2.888 & 2.828 & 2.768 & 0.0806 & 0.0857 & 0.0908 \\
0.70 & 2.328 & 2.274 & 2.224 & 0.0811 & 0.0861 & 0.0913 \\
0.65 & 1.840 & 1.800 & 1.755 & 0.0816 & 0.0867 & 0.0918 \\
0.60 & 1.430 & 1.395 & 1.358 & 0.0820 & 0.0871 & 0.0923 \\
0.55 & 1.087 & 1.055 & 1.022 & 0.0824 & 0.0876 & 0.0926 \\
0.50 & 0.802 & 0.776 & 0.750 & 0.0828 & 0.0879 & 0.0930 \\
0.45 & 0.571 & 0.550 & 0.540 & 0.0830 & 0.0882 & 0.0933 \\
0.40 & 0.398 & 0.381 & 0.365 & 0.0832 & 0.0883 & 0.0935 \\
\hline
\end{tabular}

4.1 Influence of length on the leakage of soft plunger oil pump

According to the data in Tables 1 and 2, curve fitting is performed to obtain the change curve of the oil pump leakage and soft plunger deformation with the length, as shown in Fig. 3. The figure shows that the amount of deformation increases with the length of the soft plunger, and the different initial clearances meet this rule. Under the influence of the amount of deformation, the leakage amount will change accordingly. Under the different initial clearance conditions of the soft plunger and pump barrel pair, the leakage quantity and the length of soft plunger show a negative correlation, that is, the longer the soft plunger is, the smaller the leakage quantity will be. The larger the initial clearance is, the more obvious the decreasing trend of the leakage is.

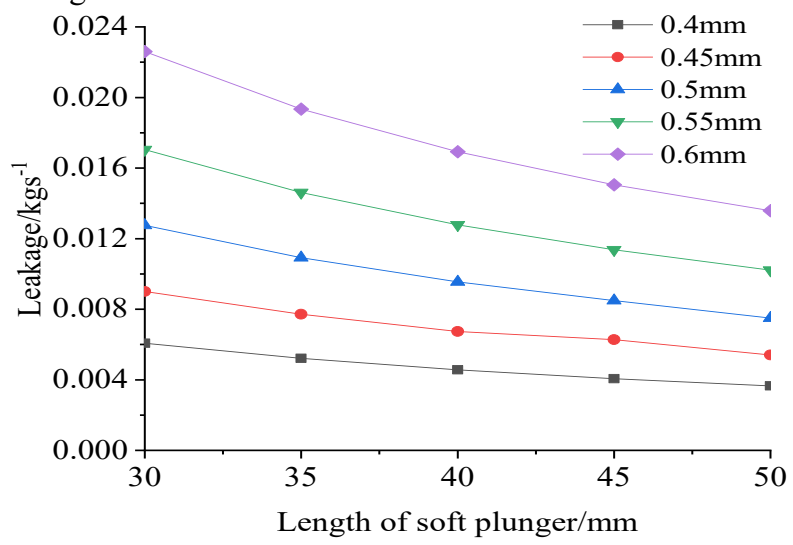

(a)

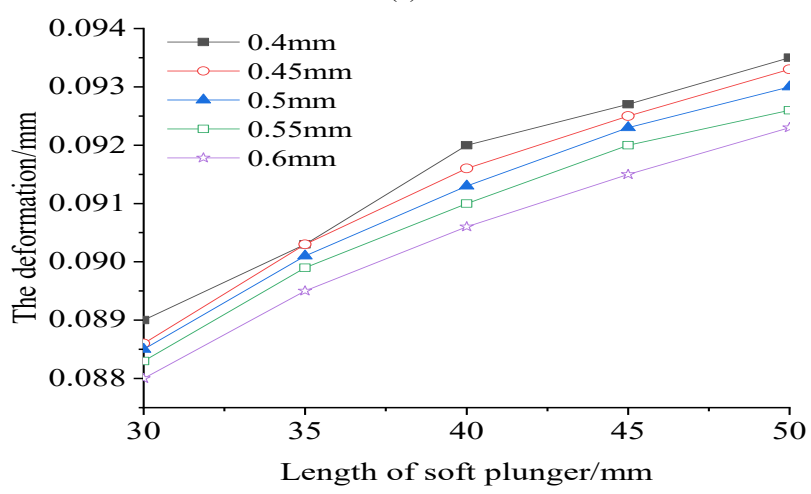

(b)

Fig. 3. Change curve of leakage and deformation with the length of the soft plunger (a) Leakage (b) Deformation
According to the data in Table 1, the fitting equation of the curve of the pump leakage with the length of the soft plunger is determined as

$f(x)=k x^{b}$

where $k, b$ are the coefficients.

The parameters of the fitting equation (Equation (2)) also vary with the initial clearance of the soft plunger-pump barrel pair, and the specific parameters are shown in Table 5.

Table 5. Fitting parameters of the relationship between the leakage and the length of the soft plunger

\begin{tabular}{l|l|l|l|l|l}
\hline \multirow{2}{*}{ coefficient } & \multicolumn{5}{|l}{ initial clearance $h_{0}\left(10^{-1} \mathrm{~mm}\right)$} \\
\cline { 2 - 6 } & 0.4 & 0.45 & 0.5 & 0.55 & 0.6 \\
\hline$k$ & 1.787 & 2.3445 & 4.1444 & 5.1318 & 6.7644 \\
$b$ & -9.9441 & -9.5913 & -10.2303 & -10.0098 & -9.9955 \\
\hline
\end{tabular}

According to the fitting equation and parameters of the curve of the change of the oil pump leakage with the soft plunger length, the parameters are nearly constant $(-1)$, which is consistent with the theoretical calculation model of Equation (1), verifying the change rule that the leakage of the multi-stage soft plunger oil pump decreases with the increase of the soft plunger length. Under the same parameter condition, if the length parameter of the soft plunger remains unchanged, changing a series of soft plungers will not affect the leakage of the oil pump.

\subsection{Influence of thickness on the leakage of soft plunger pump}

According to the data in Table 3 , the fitting equation corresponding to the curve of the leakage quantity versus the thickness of the soft plunger is

$f(x)=a e^{-x / t}+y_{0}$

where $a, t, y_{0}$ are the coefficients.

Table 6. Fitting parameters of the relationship between leakage and the thickness of soft plunger

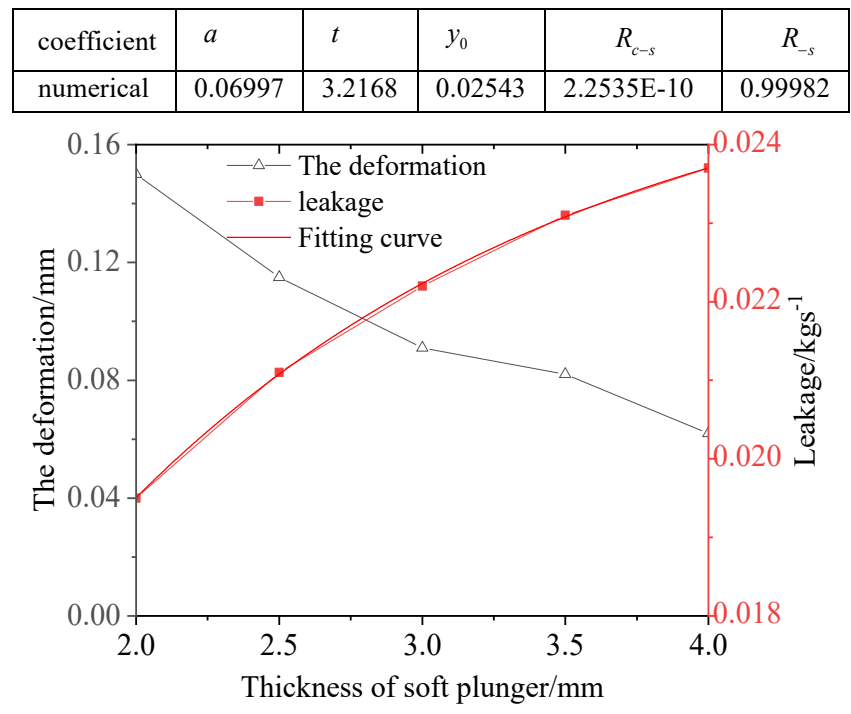

Fig. 4. Change curve of leakage and deformation with the thickness of soft plunger 
Fig. 4 indicates that the deformation of the soft plunger decreases as the thickness increases, and the leakage increases with the thickness. According to the fitting curve and equation, an exponential relationship exists between the leakage of the soft plunge-pump barrel pair and the thickness of the soft plunger.

\subsection{Effect of initial clearance on the leakage of soft plunger oil pump}

According to the data in Table 3, the fitting equation of the curve of soft plunger leakage with the initial clearance is

$$
f(x)=a x^{b}
$$

where $a, b$ are the coefficients.

Table 7. Fitting parameters of the relationship between the leakage and the initial clearance

\begin{tabular}{|l|c|c|c|c|}
\hline coefficient & $a$ & $b$ & $R_{c-s}$ & $R_{-s}$ \\
\hline numerical & 0.06997 & 3.1268 & $2.2535 \mathrm{E}-9$ & 0.99997 \\
\hline
\end{tabular}

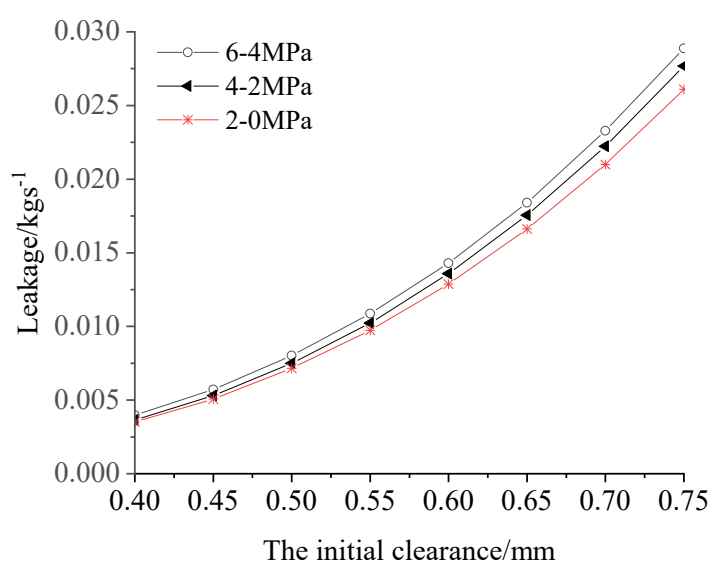

(a)

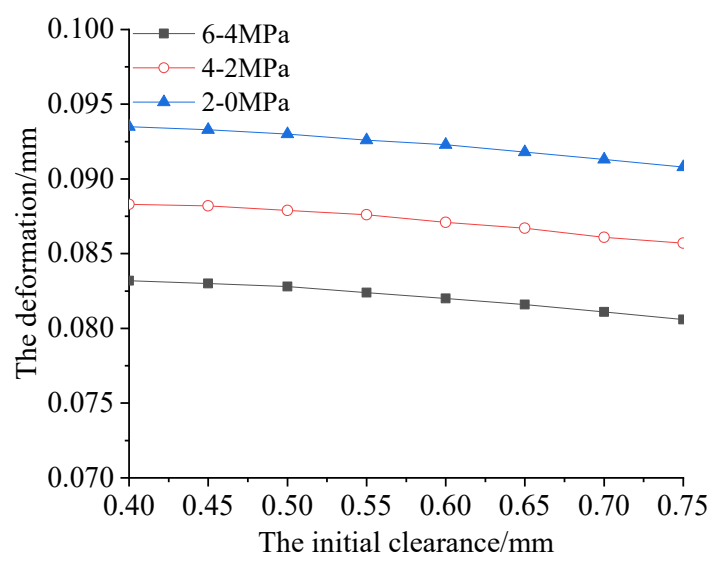

(b)

Fig. 5. Change curves of leakage and deformation with the initial clearance (a) Leakage (b) Deformation

According to the fitting curve in Fig. 5, the leakage of the oil pump increases significantly with the initial clearance between the soft plunger and pump barrel pair. According to the fitting equation, the relationship between the leakage of the oil pump and the initial clearance between the soft plunger and pump barrel pair is approximately at third power. The data in Table 4 shows that the classification calculation method is adopted to obtain the leakage of different soft plunger stages to meet the change rule, that is, the leakage of the upper stage of soft plunger is slightly larger than that of the next stage of the soft plunger, for each stage of the soft plunger to bear the same pressure difference. According to the energy conservation law of the basic theory of fluid mechanics, the initial clearance of the latter stage of the soft plunger and pump barrel pair must be slightly larger than that of the previous stage to meet the constant leakage requirement of each stage of the soft plunger.

\section{Conclusion}

A physical model for soft plunger pumps was constructed to reveal the relationship between the leakage amount and various important structural parameters, namely, length, thickness, and initial clearance. The leakage amount of soft plunger pumps was analyzed through numerical simulation and experimental investigation. The following conclusions could be drawn.

(1) A negative correlation exists between the leakage and the length of the soft plunger. After the static pressure of the outlet and inlet is determined, the reasonable length range of each stage of the soft plunger is determined by the leakage. Under the premise of satisfying the pump efficiency, the materials waste can be reduced, and cost savings can be achieved by the reasonable choice of soft plunger length.

(2) A positive correlation exists between the leakage amount of the oil pump and the thickness of the soft plunger, and the increase in thickness increases the leakage amount. A positive correlation also exists between the leakage of the oil pump and the initial clearance of the soft plunger-pump barrel pair, and the leakage of the oil pump increases obviously with the initial clearance of the soft plunger-pump barrel pair. Therefore, to ensure the inner diameter of the soft plunger is unchanged and to determine the outer diameter of the soft plunger can effectively improve the pumping efficiency of the multi-stage soft plunger pump.

(3) Under the condition of satisfying the conservation of mass from the fluid inlet to the fluid outlet, the length of the soft plunger shows a decreasing trend, the thickness shows an increasing trend, and the initial clearance between the soft plunger and pump barrel pair shows an increasing trend.

(4) Two optimization schemes, which change the length and diameter parameters of the soft plunger, are proposed, providing a theoretical basis for the structural design and parameter optimization of the multistage soft plunger of the oil pump.

Numerical simulation is combined with theoretical discusses in this study, and a new understanding of the relationship between the leakage amount of the soft plunger pumps and the main structural parameters of soft plungers is obtained. The proposed model is simple and practical and has a certain reference value for the subsequent development of soft plunger pumps. However, given the lack of on-site monitoring data, further studies should be conducted to combine monitoring data with the proposed model for a full understanding of the operation rules of soft plunger pumps under complex conditions.

This is an Open Access article distributed under the terms of the Creative Commons Attribution License. 


\section{References}

1. C, N. A., J, B. S., M, M. J., A, S. J., Tom, A., Sawson, T.,Wang, L., Yang, S. Matteo, C., F, J. T., "Readily Constructed Glass Piston Pump for Gas Recirculation". American Chemical Society omega, 5(27),2020, pp.16455-16459.

2. Kim W., Lim C., Chai J., "Development of a sdms (Self-diagnostic monitoring system) with prognostics for a reciprocating pump system". Nuclear Engineering and Technology, 52(6),2020,pp.1188-1200.

3. Gil, K. Y., Tae, K. S., Bog, H. Y., Nam, Y. S., Yeon, S. H., "System Modeling of a Bi-directional Outlet Variable Swash Plate Type Axial Piston Pump with Two EPPR Valves". Journal of Drive and Control, 17(1),2020,pp.51-60.

4. Liu, J., "New progress and development trend of oil recovery technology". Chemical Enterprise Management, (15), 2015, pp.169.

5. Ren, C., Zhu, Y., "Development and application of multistage soft plunger pump". Oil Production Engineering, (04),2020, pp.59-61.

6. Wang, Y., Ran, L., Guo, Q., "Research and application of flexible pluger \& retrievable standing valve pumps". Oil Drilling \& Production Technology, 32(2), 2010, pp.68-70.

7. Yang, X., Ma, N., Liu, Sh., "Evaluation of Application Effect of Common Lifting Process System in Strong Alkali ASP Flooding". Chemical Engineering \& Equipment, (10),2020,pp.92-94.

8. Cui, L., Chu, G., Zhang, Ch., "Appfication of soft plunger pump with hydraulic start-up in sand producing block". Special Oil \& Gas Reservoirs, 9(5),2002, pp.77-79.

9. Richardson, D., Sadeghi, F., Rateick, G. R, Rowan, S., "Surface modification effects on lubricant temperature and floating valve plate motion in an axial piston pump". Proceedings of the Institution of Mechanical Engineers, Part J:Journal of Engineering Tribology, 234(1),2020, pp.3-17.

10. Lee, B., Yu, Y., Cho, Y.-J., "Effect of piston clearance on the lubrication performance in axial piston pump". Industrial Lubrication and Tribology, 72(1),2020, pp.146-150.

11. Newton, C. H., Behnia, M., "Numerical calculation of turbulent stratified gas-liquid pipe flows". International Journal of Multiphase Flow, 26(2),2000, pp.327-337.
12. Jafri, M., Bale, J. S., Thei, A. R., "Experimental Study of Waste Valves and Delivery Valves Diameter Effect on the Efficiency of 3-Inch Hydraulic Ram Pumps:Original papers". International Journal of Fluid Machinery and Systems, 13(3),2020, pp.615-622.

13. Zhao, Y., "Development and Application of Soft Seal Piston Pump". China Petroleum Machinery, 39 (8),2011, pp.82-84.

14. Li, Q., "Design and Experimental Research of Pump with Sectionalized Soft Plunger Used in ASP Flooding".Oil Field Equipment, 43(4),2014, pp.68-70.

15. Li, J., "Study on Flexible Plunger Pumping System". Doctoral Dissertation of China University of Petroleum, China 2010, pp.22-27.

16. Gao, J., Yao, Ch., Li, S., "Mechanical Simulation Analysis of Reciprocating Pump Crosshead with Clearances in Prismatic Pair". In: Proceedings of 2019 4th International Conference on Automatic Control and Mechatronic Engineering, Chongqing,China: ACME, 2019, pp.29-36.

17. Kireev, S. O., Nikishenko, S. L., Vasil'ev, B. N., Vasil'ev, M. A., "Analysis of Operating Conditions of Sliding Friction Units of Driving System of high-pressure oil and gas well service plunger pumps". Chemical and Petroleum Engineering, (52),2016, pp.332-338.

18. Ham, J., Kim, S., Oh, J., Cho, H., "Theoretical investigation of the effect of a relief groove on the performance of a gerotor oil pump". Jour nal of Mechanical Science and Technology, 32(8),2018, pp.3687-3698. 19. Medvedovski, E., Jiang, J. R., Robertson, M., "Tribological properties of boride based thermal diffusion coatings". Advances in Applied Ceramics, 113 (7),2014, pp.427-428.

20. Yu, M., "Research on Model of pump's working Performance". China University of Petroleum, Master thesis of China University of Petroleum, China, 2013, pp.45-46.

21. Jiang, F., "The Impact of Pump Barrel-piston Clearance on the Leakage of Pump in High Temperature Deep Well". China Petroleum Machinery, 44 (06),2016,pp.102-105. 